\title{
LE DELTA DU DANUBE ET SES RICHESSES
}

\author{
par George BARCA \\ Ingénieur, Docteur, Agrégé \\ Professeur Polytechnique de Galatzi -- ROUMANIE
}

Le Delta du Danube, contrée d'un étrange pittoresque, a attiré dès les temps les plus reculés l'attention des voyageurs poussés vers les rivages lointains du vieux fleuve, soit par le hasard, soit par des intérêts de commerce ou des actions militaires, ou bien simplement par un besoin de connaitre de nouveaux horizons.

Dès l'époque reculée d'Hérodote, les voyageurs revenant des rivages du Pont Euxin, où florissaient les nombreuses cités de la Grèce antique, ne tarissaient pas de vanter les riches contrées du nord de la Scytie, là où, disaient-ils, le fleuve Istros se jetait dans la mer par des dizaines de bras.

Ils évoquaient, comme un conte, les richesses de ces terres et de ces eaux où des nuées de poissons peuplent les lacs tapis dans la forèt de roseaux et où le vin abondant et le miel adoucissaient la vie si dure des habitants des bouches du Danube.

A l'exception des anciennes cités, dont les vestiges montent encore la garde sur les falaises de la Dobroudja et sur lesquels le temps a irréversiblement passé sa patine, pour le reste certaines appréciations seraient valables aujourd'hui encore.

Le Delta, la partie la plus jeune du Danube, là où ce fleuve sépare ses eaux en trois bras puissants, présente aujourd'hui encore son aspect de jadis une mer de roseaux touffus, ondulant au souffle de la brise marine, où s'entrecroisent de larges canaux menant vers des lacs foisonnant de toutes les espèces de poissons. Le Delta est, aujourd'hui comme jadis, le paradis des oiseaux migrateurs revenant des tropiques et l'animant du coloris fascinant de leur plumage. Des saules noueux et tordus balayent l'onde de leurs rameaux et veillent, comme jadis, sur ces canaux, leur conférant une poésie et marquant leurs sinueux contours dans cette mer de roseaux. Tout est presque pareil au passé, mais la présence de l'homme est plus fréquente aujourd'hui dans ces parages, qui semblaient réservés aux oiseaux migrateurs, à la multitude des poissons et aux martres à la fourrure fauve, se repaissant de cette proie ignorante et facile à saisir. 
En hiver, lorsque le blizzard de l'immense steppe russe se rue sur le Delta, couvrant le miroir des eaux d'une épaisse couche de glace, les bras du Danube semblent de vastes boulevards figés, bordés de vieux saules qui tordent leurs branchages nus au-dessus des rivages.

En cette saison, les canots dorment au bord de l'eau et les habitants de ces parages parcourent les chemins du Delta à pied. Ils vont et viennent sur le miroir glacé des canaux et des lacs endormis, attendant la fin du sommeil hivernal enchanté.

Devant ces images, notre pensée s'évade vers les âges révolus, lorsque les cruels Sarmates, recouverts de lourdes cuirasses, s'élançaient à cheval sur le Delta glacé, semant l'épouvante parmi les braves légionnaires romains, qui veillaient sur la Scytie Mineure de jadis - Dobroudja d'aujourd'hui.

Mais, le dégel mettait fin à ces incursions venant des steppes lointaines du soleil levant. Les eaux revenaient de leur torpeur, le Delta du Danube sortait du) grand silence hivernal, réveillé par une autre invasion. Cette fois la nuée des oiseaux envahissait le Delta, le couvrant d'un bout à l'autre de leur jacassement, de leur pépiement, d'un frémissement aưquel n'aurait pu résister aucune légion du monde.

Venant du Nord glacé, ainsi que des chaudes contrées du Nil, le plumage multicolore de ces hôtes egaye le printemps du Delta. Pendant ces mois d'éveil de la nature, lorsque le Delta redevient le paradis des oiseaux, les grandes eaux du Danube apportent maintenant de la Forêt Noire et du Centre de l'Europe un limon fertile, qu'elles déposent dans les eaux saumâtres devant les trois bras du Danube. Grâce à ces dépôts, la superficie de la Roumanie gagne chaque années presque 60 hectares sur la mer.

Voici le moment où l'innombrable foule des poissons s'éveille de son sommeil hivernal. Sur la végétation palustre qui revient à la vie, les eaux du Danube, pareilles à un immense tapis, avancent : du plateau de la Bessarabie, jusqu'à celui de la Dobroudja, d'un bout à l'autre du Delta jusqu'à la mer, tout n'est qu'une nappe d'eau. La présence de la terre se révèle seulement par les saules bordant les canaux et les lacs.

Au printemps, lorsque les grandes eaux recouvrent le Delta, les pêcheurs vont sans entrave dans leurs canots d'un village à l'autre par des raccourcis. C'est la phase dynamique et créatrice du Delta, car les poissons et les oiseaux déposent leurs œufs soit sur l'herbe des pâturages encore ruisselants d'eau, soit dans des nids bâtis hâtivement dans les roselières et les branchages des saules.

Par les trois bras du Danube apparaissent les majestueux esturgeons; fuyant la Mer Noire, ils remontent les eaux douces pour y pondre leurs ceufs : le précieux caviar. Aussitôt suivent les harengs à la chair savoureuse; ceux-ci devenant adultes dans les eaux marines, reviennent pondre leurs œufs dans les eaux douces du Danube, probablement pour ne pas faillir à l'attraction de leur origine, à l'instar des esturgeons. Dans cette saison, le barrage des filets sans nombre les guette au long du Danube et l'odeur piquante de leur chair savoureuse inonde, pour une période assez brève, le marché.

Ensuite les eaux du vieux fleuve se retirent dans leur lit ancestral et le Delta prend l'aspect d'une gigantesque * pampa * aquatique, coupée de langues de terre, où de nombreux troupeaux broutent l'herbe grasse des pâturages. 


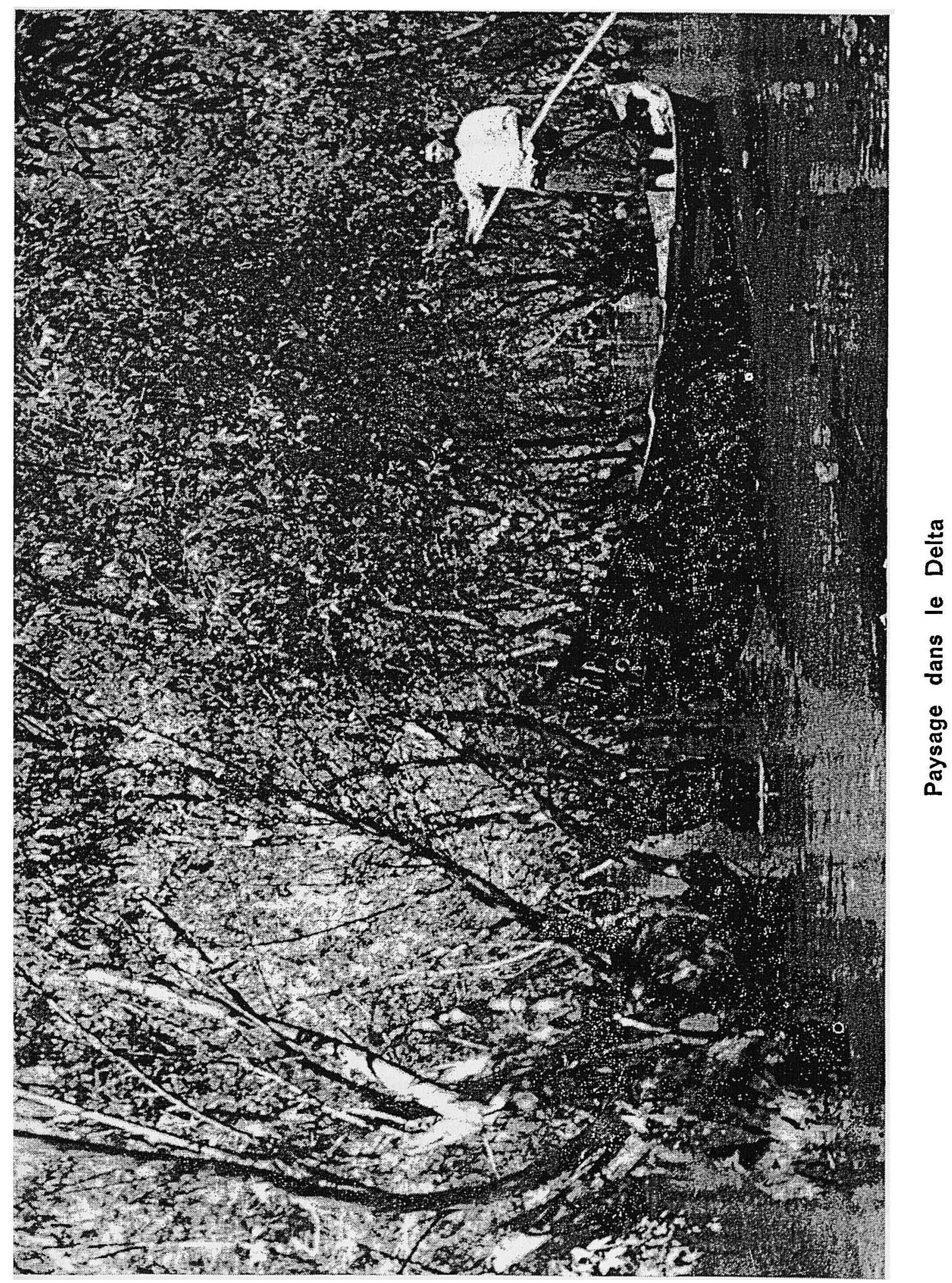


Et voici l'été. Fatigué de l'effort du printemps, le Delta du Danube semble s'assoupir sous la chaleur torride du soleil miroitant dans les eaux immobiles des lacs. Jusqu'à la fin de l'été, le Delta est une contrée de rêve pour tous ceux qui sont en quête de repos, d'abondance, de rêverie.

Mais lorsque l'automne approche et que le feuillage prend une teinte vieil or, le Delta offre tout son potentiel d'énergie accumulée au cours de l'année.

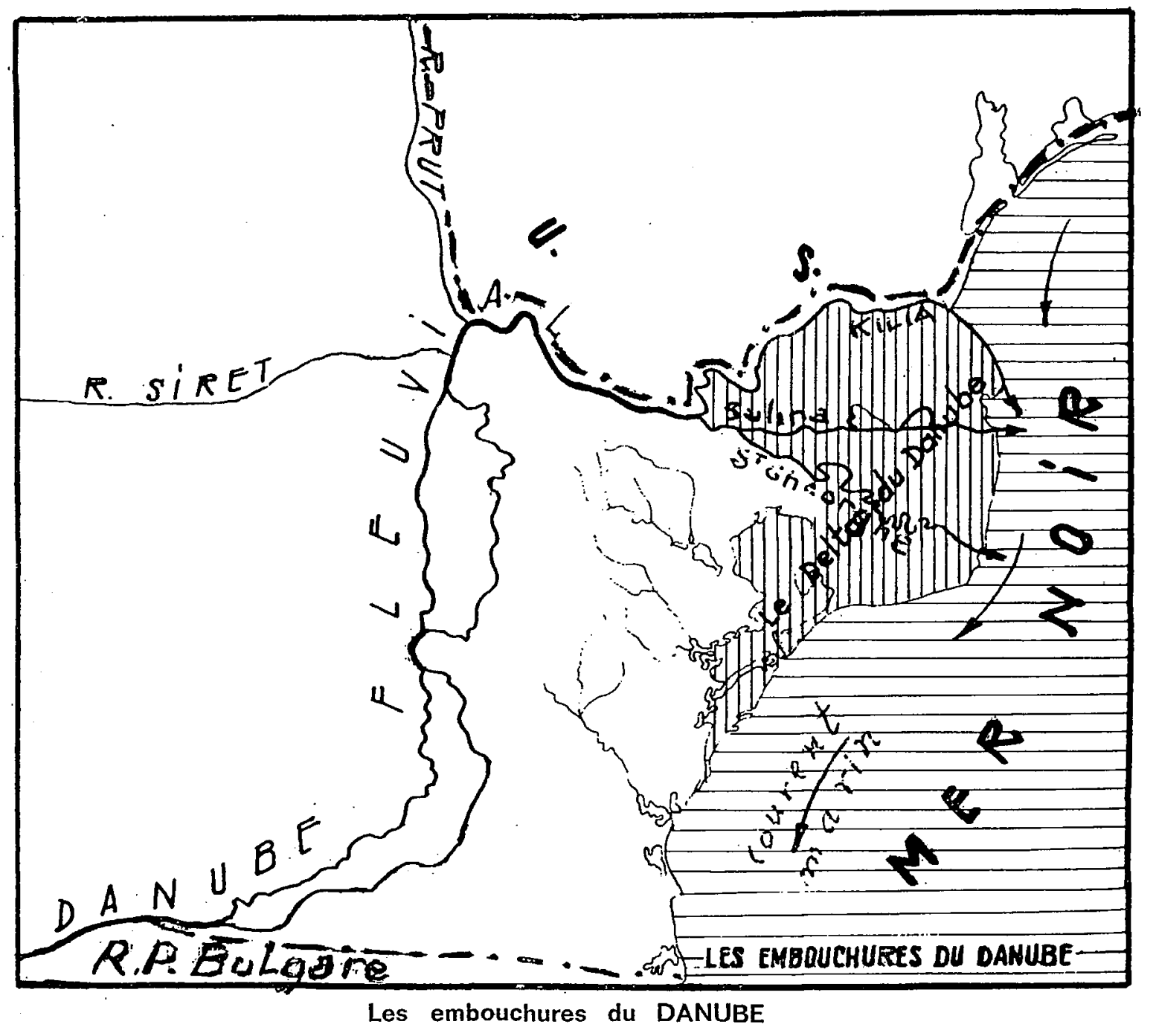

Contemplé d'un avion, il semble un immense pain bien doré, croustillant, que la nature offre à l'homme pour le récompenser de ses efforts. C'est le moment de récolter les fruits des vergers bordani les rivages des bras du Danube, des vignobles plantés sur les bancs de sable et la foule de poissons dorés se dirigeant vers les endroits où ils passeront leur sommeil hivernal.

Bientôt les eaux seront figées par le gel; les hommes, dans le fracas assourdissant de leurs machines, envahiront le Delta pour la récolte du roseau qui durera jusqu'au printemps. C'est le don le plus précieux du Delta à l'économie du pays.

De nombreux ouvrages mentionnent l'existence de cette contrée aussi riche que mystérieuse, aujourd'hui comme jadis ; mais les informations scientifiques concernant le Delta remontent à 100 ans à peine. Pour la plupart, elles sont dues au savant roumain Grigore Antipa $(1868-1944)$, biologue d'une haute tenue scientifique. 
Les premiers travaux d'hydro-améliorations ayant pour but d'améliorer les conditions biologiques des poissons et du roseau, sont l'œuvre de ce savant et de-ses collaborateurs.

Son œuvre a été continuée par ses élèves et par les nouvelles générations d'ingénieurs. Ceux-ci ont dressé un programme plus complexe de travaux concernant non seulernent la mise en valeur du Delta du point de vue économique, mais aussi du point de vue touristique, car cette intéressante région de la Roumanie renferme un important potentiel dans ce domaine.

Pour ceux qui viennent connaitre ici non seulement le pittoresque, mais aussi les secrets des eaux dormant depuis des millénaires dans cette oasis des lagunes du vieux Danube, le Delta est un vaste laboratoire; bon nombre des étranges processus de la vie aquatique peuvent y trouver une réponse satisfaisante pour le scientifique passionné par l'inconnu encore indéchiffrable de ce vaste organisme deltaĩque, unique au monde en son espèce.

Les récentes recherches nous font savoir que les commencements du Delta du Danube remontent au $12 \mathrm{e}$ millénaire.

Quelle que soit l'époque à laquelle il a fait son apparition, une chose est certaine : c'est que le Delta a pris naissance par l'action combinée des mouvements techtoniques et des agents qui ont réussi à lui imprimer la morphologie actuelle; les alluvions du Danube combinées à l'action des vagues et des courants marins y détiennent le rôle principal.

Il y a environ 5000 ans, le Delta était un golfe de la Mer Noire, où le Danube jetait ses eaux calmes. Au fur et à mesure, les dépôts alluvionnaires ont divisé la nappe ininterrompue de l'eau en de grands lacs deltaĩques. Dans cette division du golfe, l'action du fleuve domine dans la zone occidentale du Delta, la terre ferme étant d'origine alluvionnaire. Dans la zone orientale, l'action du fleuve s'entrepénètre avec celle de la mer, qui manifeste sa contribution à la génèse du Delta par de nombreux cordons littoraux, formés à la suite des millénaires oscillations du niveau de la Mer Noire. A ce processus purement mécanique, déterminé par l'action de l'eau et complété par l'action saline, un processus biologique s'est superposé; ce dernier détient actuellement un rôle prioritaire dans l'évolution de cette zone où se jette le Danube.

Du point de vue morphologique, le Delta du Danube se divise en deux grandes zones:

- la zone comprise entre les deux bifurcations et les cordons littoraux Letea et Caraorman, d'origine fluviale;

- la zone comprise entre les cordons mentionnés et le littoral de la Mer Noire, dans laquelle la terre a subi tout particulièrement l'influence de la mer. Dans cette action dynamique du Danube, les alluvions qu'il ramasse sur son trajet de $2800 \mathrm{~km}$ (évaluées à environ 75000000 tonnes par an (1), ont été assez faibles au début, mais elles ont pris une importance croissante au long des siècles, surtout à la suite du défrichement des forêts; toutefois, la masse de ces alluvions a été suffisamment grande pour colmater la dépression du golfe deltaĩque.

(1) D'après les mesures effectuées par l'ingénieur Hartley, dans le cadre de la Commission européenne du Danube (C.E.D.) 
Par les trois bras du Danube, l'espace deltaĩque a été divisé en deux grands bassins, nommés : l'île Letea au nord, et l'île St-Gheorghe au sud.

Des trois bras du Danube : Kilia, Sulina et St-Gheorghe, ce dernier est supposé le plus ancien et Kilia le plus jeune.

L'intense vitalité du bras Kilia, manifestant la tendance de créer un delta propre, est une preuve de son âge récent.

La proportion des débits transportés par les trois bras est environ la suivante : Kilia $68 \%$, Sulina $12 \%$. St-Gheorghe $20 \%$. (2)

Par l'effet des grands débits d'eau véhiculés par le bras Kilia, celui-ci avance vers la mer d'environ $70 \mathrm{~m}$ chaque année, ajoutant ainsi au Delta une superficie de près de 65 ha/an. (3) L'apport des autres bras est beaucoup plus lent. C'est ainsi qu'avec de faibles écarts, le bras St-Gheorghe n'a plus avancé depuis près de 600 ans et Sulina depuis près de 1000 ans. II parait donc que nous nous trouvons à l'époque d'une forte ascension du bras Kilia, demeuré auparavant en retard sur les deux autres. En conclusion, l'avancement du Delta n'est pas simultané sur tout son trajet, s'effectuant tantôt par un bras, tantôt par l'autre, mais réussissant tout de même à maintenir sa direction nord-sud.

Le rapport entre la terre et l'eau diffère d'une année à l'autre et d'une saison à l'autre. Voici un aperçu du rapport terre/eau :

$\begin{array}{ll}\text { - eaux basses } & 1 / 2 \\ \text { - eaux moyenes } & 1 / 5 \\ \text { - eaux hautes } & 1 / 9 \\ \text { - eaux exceptionnellement hautes } & 1 / 13\end{array}$

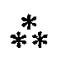

Le Delta est un organisme vivant, par lequel le fleuve tâtonne la voie la plus indiquée pour écouler ses eaux vers la mer, en fonction des possibilités de celle-ci : niveau, courants marins etc.

L'évolution du Delta, dans le temps et dans l'espace, a lieu par le colmatage des zones en amont et par le gain de nouveaux terrains dans la zone du littoral. Voilà pourquoi le Delta du Danube se présente comme deux sous-régions géographiques distinctes : le Delta proprement dit - formation fluviale prédominante comprise entre les bras Killia, Sulina et St-Gheorghe, représentant sur le territoire roumain une superficie de $3430 \mathrm{~km}^{2}$, et le Delta lagunaire (Razelm-Sinoe) environ $990 \mathrm{~km}^{2}$, formé à la suite de la séparation du golfe existant des eaux marines par un cordon littoral de sable.

La lagune Razelm-Sinoe est alimentée par les eaux du Danube à travers des canaux naturels et artificiels; l'eau de la mer y pénètre aussi par les brèches existant dans le cordon littoral, de sorte que la salinité croit progressivement vers le sud dans ses compartiments, allant de $1 \mathrm{~g} / \mathrm{l}$ dans son compartiment nord (Razelm) jusqu'à une salinité supérieure à celle de la Mer Noire, à son extrémité sud (Sinoe).

Dans le Delta proprement dit, le régime hydrologique se trouve sous l'influence directe des niveaux du fleuve, tant en ce qui concerne les possibilités de changer l'eau dans les lacs par de l'eau fraiche, que pour la dynamique de leur colmatage alluvionnaire.

(2) GRIGORE VASILESCU : Contributions à l'étude de la formation du Delta du Danube.

(3) ION VIDRASCU : La mise en valeur de la région inondable du Danube. 
Les superficies de terre ne détiennent pas un coefficient important dans le Delta du Danube; elles sont, du reste, périodiquement inondées par les grandes eaux du fleuve se confondant alors avec les zones des marais qui détiennent environ $70 \%$ de la superficie du. Delta fluvial.

La zone des marais proprement dite est au premier coup d'œil une mer de roseau parsemée de lacs - de larges miroirs - parfois de 3000 à 5000 ha; leur profondeur varie de 2 à $5 \mathrm{~m}$. Le fond des lacs est couvert de boue sapropélique, d'une épaisseur de 1 mètre, dont le potentiel trophique peut assurer des productions importantes et permanentes, par alimentation en eau fraiche du Danube.

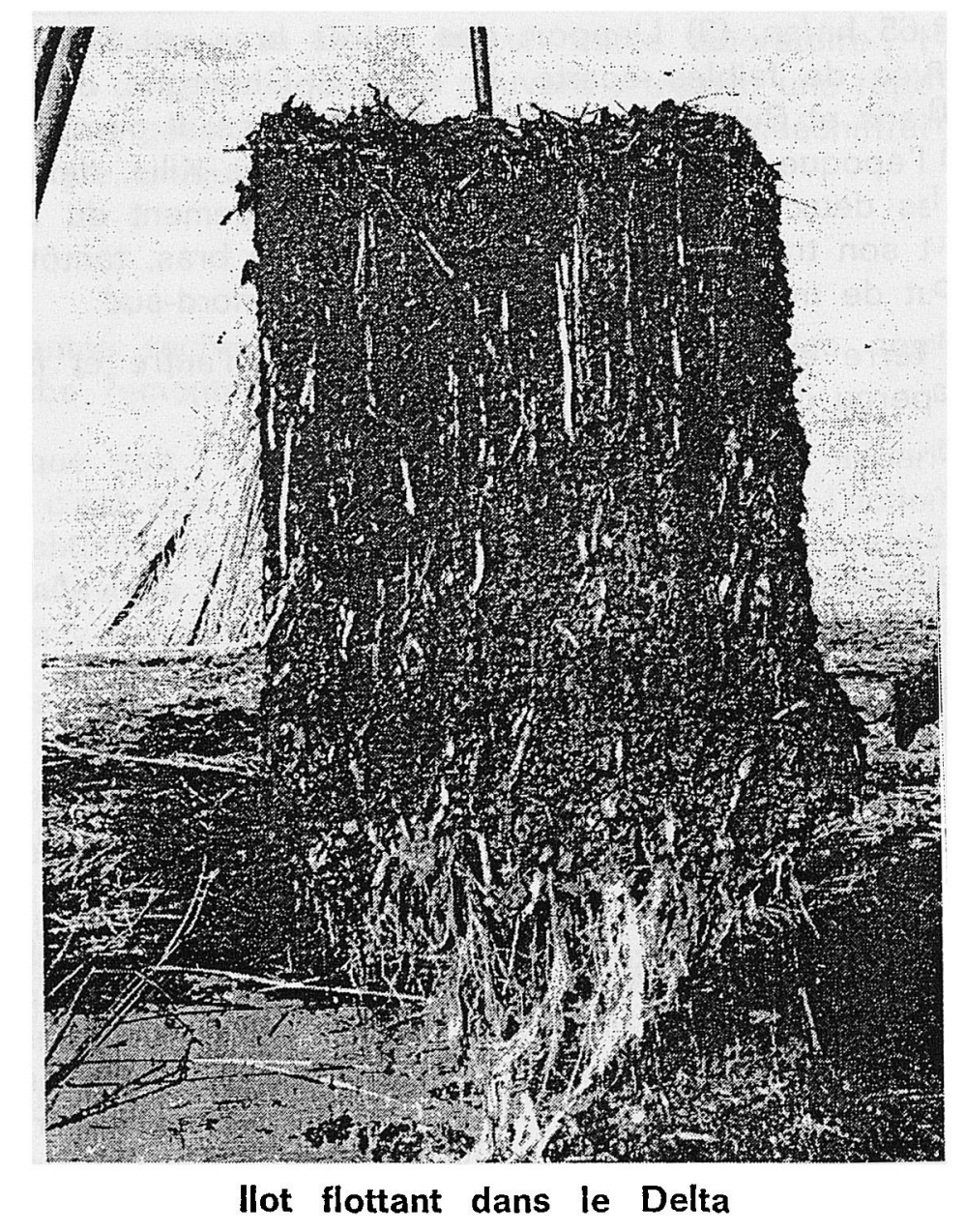

Les différentes utilisations du Delta s'entre-pénètrent en général. Les superficies sèches sont destinées surtout à l'agriculture, servant aussi à la zootechnie, à la viticulture et à la cutlure des arbres fruitiers.

Quant aux terrains arables, là où les circonstances favorables ont permis leur aménagement, on a réussi à gagner des terres au cœur du Delta même. De nombreux terrains inondables, envahis de roseaux ont été endigués et se sont transformés en de vastes labours et cultures horticoles.

Les terrains couverts de pâturages sont utilisés au printemps alternativement pour l'élevage du bétail et pour l'économie piscicole, servant à la reproduction naturelle des poissons, en fonction des oscillations du niveau des eaux qui les recouvrent ou les laissent libres.

Par endroits, sur les terrains du Delta il y a des zones massives de forêts de saules, regénérées spontanément ou des plantations de peuplier canadien. En 
dehors des forêts, qui apportent des bénéfices au secteur sylvicole, il y a sur certaines dunes de sable, de provenance marine, des forêts déclarées monuments de la nature. Surgies spontanément, celles-ci sont un mélange hétérogène de chêne et d'essences tendres (peuplier, aulne etc.), enchevêtrés de lianes à l'allure exotique; c'est un décor étrange où le vert foncé de la forêt contraste vivement avec le blanc éblouissant de ces dunes de provenance marine.

La zone des marais du Delta représente une superficie de 290000 ha couverte de roseau fixé sur le sol. A côté de ces roselières, il y a aussi des îlots flottants (roseau associé aux plantes xérophiles) qui voguent au gré du vent sur le miroir des eaux. Ces ilots flottants couvrent une superficie d'environ 20000 ha et leur tendance' est d'accaparer horizontalement toute la surface des lacs où ils font leur apparition; par leur croissance sur la verticale ils tendent à toucher le fond de ces bassins, arrivant à les transformer, à les annihiler progressivement; finalement, il n'est plus possible de s'en débarrasser.

Le roseau de cette région présente un aspect très dense, c'est le plus compact au monde, et une grande richesse naturelle de la Roumanie.

Par sa teneur en cellulose, le roseau du Delta du Danube peut remplacer le bois dans l'industrie du papier, il est du reste employé à différentes autres fins de l'économie locale.

Les travaux pour l'exploitation rationnelle du roseau sont encore dans une phase expérimentale, étroitement liée au choix de l'agrégat récolteur ayant le plus grand rendement et ne devant pas nuire à la biologie de ce végétal.

Les lacs du Delta du Danube sont du type eutrophe, caractérisés par un développement abondant des plantes immergées.

La production piscicole du Delta repose sur des espèces de valeur économique, telles que carpe, brème, sandre, silure chat etc ... ainsi que sur d'autres espèces de moindre valeur, parmi lesquelles dominent le brochet, la perche etc.

En fonction du pourcentage des rapaces, le volume de la production varie. On obtient les plus grandes productions lorsque les conditions propres au développement de la carpe coincident avec une diminution de la proportion des rapaces.

C'est le complexe de la lagune Razelm-Sinoe, dont la salinité est supérieure à celle des lacs du Delta proprement dit, qui offre des conditions tout à fait favorables à l'économie piscicole et lui impriment un caractère tout particulier.

Dans cette unité, c'est le compartiment nord, Razelm, qui détient la plus grande production piscicole, représentant plus de $50 \%$ de la superficie du complexe de la lagune toute entière.

Les espèces dominantes sont la carpe, la brème et la sandre. La production de ce compartiment est d'autant plus élevée que la salinité tend vers $1 \mathrm{~g} / \mathrm{l}$ d'eau. On a constaté qu'une baisse de la salinité au-dessous de $0,5 \mathrm{~g} / \mathrm{l}$ entraine la pénétration de certaines espèces d'eau douce indésirables, par l'intermédiaire des canaux alimentant la lagune avec les eaux douces du Danube. Grâce à elles, la salinité propice de cette lagune varie de 1 à $3 \mathrm{~g} / \mathrm{l}$; elle résulte d'une action hydrotechnique dirigée qui règle l'admission des eaux douces du Danube et celle des eaux salées de la Mer Noire.

Dans la zone sud du complexe lagunaire Sinoe, par les brèches du cordon de sable, pénètre le mulét (Mugil auratus) qui trouve là des conditions exceptionnelles de nourriture. Dans ce secteur, la salinité est bien plus forte que dans la zone nord, dépassant $8 \mathrm{~g} / \mathrm{l}$; dans bien d'autres secteurs, la salinité est supérieure à celle de la Mer Noire.

(à suivre) 